\title{
A Rat Embryo Staging Scale for the Generation of Donor Tissue for Neural Transplantation
}

\author{
E. M. Torres,* U. M. Weyrauch,* R. Sutcliffe, $\dagger$ and S. B. Dunnett* \\ *Department of Biosciences, Cardiff University, Cardiff CF10 3US, UK \\ $†$ Charles River Laboratories, Research Models \& Services, UK
}

\begin{abstract}
In rat models of Parkinson's and Huntington's diseases, embryonic neural cells obtained from embryos of specified ages can be implanted into the brain to partially restore both physiology and function. However, in litters produced using overnight mating protocols (often from commercial suppliers), the embryonic age can be difficult to determine precisely. As a result, embryonic size based on crown to rump length (CRL) is usually a more reliable method of embryo staging than the day of mating. This approach is not without difficulty. There are a number of rat staging scales in the literature, none of which deal with donor ages younger than E13, and there are discrepancies between scales at some donor ages. In the present article, we have devised a short mating-period protocol to produce precisely aged embryos. We show that CRL is a highly accurate, reproducible index of donor age and we present an updated embryonic staging scale for Sprague-Dawley (CD) rats that includes donor ages younger than those previously reported.
\end{abstract}

Key words: Dopamine graft; Donor age; Embryo staging

\section{INTRODUCTION}

Few laboratories have facilities large enough to allow for regular breeding of rats for the purposes of procuring embryonic tissues, and instead rely on the purchase of time-mated rats from commercial suppliers. Pregnant rats obtained in this way are produced from the pairing of dams and sires for periods of up to $24 \mathrm{~h}$, and the precise timings of estrus, mating, and conception are unknown. As a result, the ages of embryos obtained in this way cannot be determined with greater accuracy than $\pm 12 \mathrm{~h}$.

Where a more accurate determination of embryonic age is required, developmental landmarks such as somite numbers, limb buds, toe rays, and limb length can be used, although this requires some level of knowledge and expertise in the field of embryonic development (3). A much simpler method relies on measurement of the overall size of embryos to determine embryonic age. The conventional method is the measurement of crownrump length (CRL), for which size-for-age scales are available in the literature. However, this method is not without its limitations. A number of scales have been published $(1,3,4,8)$, but a comparison between these reveals discrepancies in estimates of embryonic size, par- ticularly at ages below E13 postcoitus. A further problem is that reporting of ages of donor embryos in the neural transplantation literature is inconsistent. Some authors report embryonic age from the day of coitus, others from the day the vaginal plug is observed, and some even fail to specify which of the two conventions is being used.

Where tissues obtained from embryos are to be used for transplantation, for the generation of cell cultures, or for the isolation of stem cells, it may be important to collect tissues within a specific and relatively short time window of development. Where this is the case, embryos obtained via the routine methods used by the commercial suppliers are likely to be unsuitable. Additionally, many workers need to collect embryos at the beginning of the working day, and where whole-day embryonic ages (e.g., E10, E11, E12) are required, the pairing of sires and dams would need to be carried out at the same time of day. There is a need, therefore, for mating protocols that allow the time of mating to be more accurately determined, and a corresponding embryo staging scale that can be used to accurately age embryos based on physical measurements. The present study seeks to address both of these issues. 


\section{MATERIALS AND METHODS}

Experimental Animals

All experiments were conducted in accordance with requirements of the UK Animal (Scientific Procedures) Act 1986. Twenty-four pregnant Sprague-Dawley (CD) rats were used as the source of donor embryos. Dams were supplied by Charles River UK using a mating protocol that enabled the time of mating to be accurately established. Briefly, all animals were housed under standard conditions with free access to food and water under standard 12-h light/12-h dark (0700 to 1900 h) lighting. Normally at Charles River UK, to facilitate timed mating, the estrous cycle of female rats is monitored daily between 1300 and $1500 \mathrm{~h}$ using a vaginal impedance meter, which enables identification of rats in the proestrous stage of the cycle (15). However, for the current study, on the day of mating, 60-70-day-old females were separated from the colony without impedance measurement and paired with stud males at a male/female ratio of 1:1, between 0700 and $0900 \mathrm{~h}$. The day of mating was denoted as day zero for staging purposes. After pairing, females were removed and checked for the presence of a vaginal plug. Plugged females were transported to the laboratory in Cardiff 7 days later (aged E6).

\section{Extraction of Embryos}

Pregnant dams were sacrificed at postcoitus ages of: $\mathrm{E} 11(n=3), \mathrm{E} 12(n=4), \mathrm{E} 13(n=4), \mathrm{E} 14(n=4), \mathrm{E} 15$ $(n=4)$, E16 $(n=3)$, and E17 $(n=3)$. All litters were collected between 0900 and $1000 \mathrm{~h}$, thus enabling accurate estimation of embryonic age to within $3 \mathrm{~h}$. Removal of the embryos from the pregnant mother was performed using a protocol described previously (4). Briefly, dams were terminally anesthetized by IP injection of pentobarbital, after which the uterus was surgically removed. Each embryo was carefully excised from the uterus, removed from the amniotic sac, and detached from its placenta. Where litters contained an occasional obviously underdeveloped "runt" embryo it was removed from the study. Collected embryos were placed into a petri dish containing Hank's balanced salt solution (HBSS). The number of embryos in each litter was counted and recorded. Next, each embryo was individually photographed, using a Magnafire digital camera (Optronics UK) attached to a Wild M8 binocular zoom macroscope. Embryos were photographed lying on one side, without compression or stretching in sufficient HBSS to just cover the embryos. A millimeter scale was photographed at the same time as each litter, at the same magnification. The corresponding scale was then used to measure the CRL of each embryo from the photographs (measuring the maximum length from the crown of the head to the base of the rump). Following photography, embryos were fixed and stored in a solution of $4 \%$ paraformaldehyde in $0.1 \mathrm{M}$ phosphate-buffered saline (PBS), $\mathrm{pH} 7.4$, for a minimum of 6 weeks, after which they were rephotographed and measured to determine the effects of fixation on embryo size.

\section{RESULTS}

Figure 1 is a photomicrograph of representative embryos for each of the embryonic ages studied. The mean CRLs for each donor age, together with maximum and minimum embryo sizes, are given in Table 1.

Figure 2 shows the plot of these data and forms the basis of a new rat embryo staging scale. Between the ages of E11 and E17, the CRL of CD rat embryos increased in a linear fashion at a rate of approximately $2.5 \pm 0.25 \mathrm{~mm} /$ day. The variation in CRL between litters using a 2-h mating was very small and comparable to that seen within litters (Fig. 3). To determine the effect of litter size on embryo size, the number of embryos in each litter was plotted against a relative index of embryo size. This was calculated as the difference between the mean CRL of individual litters and the mean CRL for all litters of the same age (Fig. 4).

A comparison of the new staging scale with others in the literature is shown in Figure 5. For a given embryonic age, CRL estimates are generally smallest on the scale published by Butler and Juurlink (3), and largest on that published by Ambrus et al. (1). The present scale is largely in agreement with that of Butler and Juurlink (3) from ages E11 to E13 and with that of Dunnett and Bjorklund (4) at older ages.

The effect of fixation on embryo size was small. Embryos were remeasured after fixation for at least 6 weeks in a neutral formaldehyde solution. Analysis of variance indicated that there were significant differences between fresh and fixed embryos $[F(13,288)=1.96, p<0.05]$. However, significant postfixation changes in CRL were only demonstrable at E12 and E16 (post hoc $t$-tests; $p<$ 0.001) (Fig. 6).

\section{DISCUSSION}

In published reports of experiments using embryonic rat neural tissues, there is inconsistency in the reporting of the age of the donor embryos used. As a result, it is difficult to compare experiments between laboratories or to reproduce experiments precisely. This is due to a number of factors. Firstly, conventional rat mating protocols usually involve overnight pairing of the male and female for periods up to $24 \mathrm{~h}$. At our supplier, Charles River, the procedure used to produce time-mated pregnant rats is typical of that used by most commercial suppliers: on week days, the pairing of dams and sires for mating is carried out at the end of the working day $(1500-1600 \mathrm{~h})$ and separated the following morning 


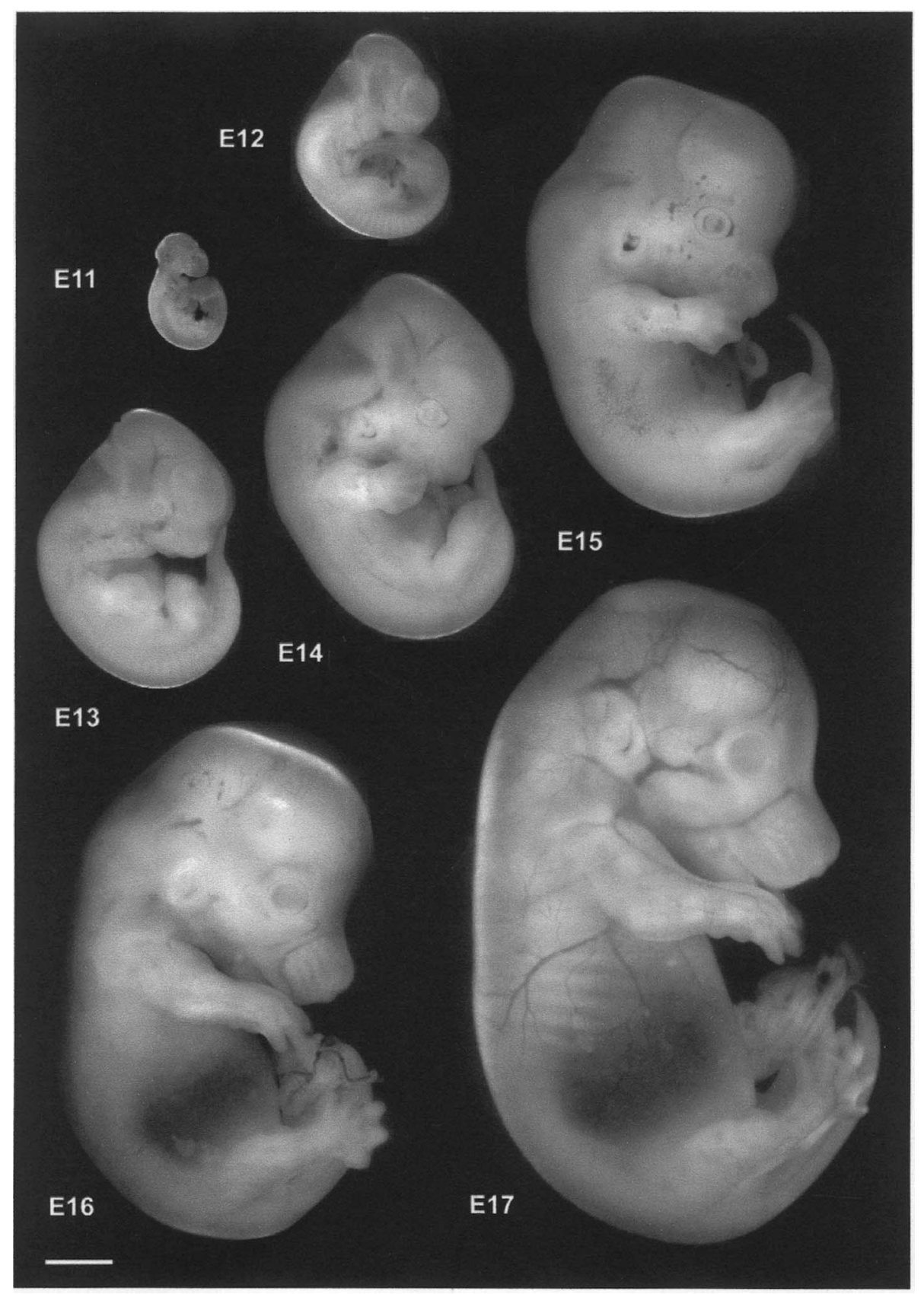

Figure 1. Photographic representation of the rat embryonic staging scale. Representative embryos from E11 to E17 postcoitus. Images are of freshly dissected embryos as photographed for measuring purposes. Scale bar: $2 \mathrm{~mm}$.

(0800-0900 h). However, on the weekend pairing is carried out in the morning $(0800-0900 \mathrm{~h})$ and pairs are not separated until the following day at the same time $(0800-0900 \mathrm{~h})$. Thus, the period of pairing varies from 17 to $24 \mathrm{~h}$, during which mating and fertilization may take place at any time. As a result, the age of the embryos produced in this way cannot be estimated with any precision. Indeed, depending on the day of pairing, estimates of embryonic age might be inaccurate by as much as $24 \mathrm{~h}$.

A second confounding factor is the common use of two conventions to denote embryonic age. In the first convention, day zero is denoted as the day of pairing; in the second convention it is denoted as the day following overnight pairing, at a time when the vaginal plug is observed. Additionally, because the pairing of sires and 
Table 1. Characteristics of Each Donor Age

\begin{tabular}{lccccc}
$\begin{array}{l}\text { Embryonic } \\
\text { Age (Days) }\end{array}$ & Mean CRL $(\mathrm{mm})$ & Minimum & Maximum & Range & Carnegie Stage \\
\hline E11 & 3.42 & 3.3 & 3.7 & 0.4 & 12 \\
E12 & 5.90 & 5.8 & 6.1 & 0.3 & 14 \\
E13 & 8.07 & 7.0 & 8.9 & 1.9 & 16 \\
E14 & 10.52 & 10.2 & 10.9 & 0.7 & 18 \\
E15 & 12.96 & 12.9 & 13.0 & 0.1 & 20 \\
E16 & 15.53 & 15.1 & 16.4 & 1.3 & 22 \\
E17 & 18.41 & 17.4 & 19.8 & 2.4 & 24 \\
\hline
\end{tabular}

CRL: crown-to-rump length. Carnegie stages calculated from Butler and Juurlink (3).

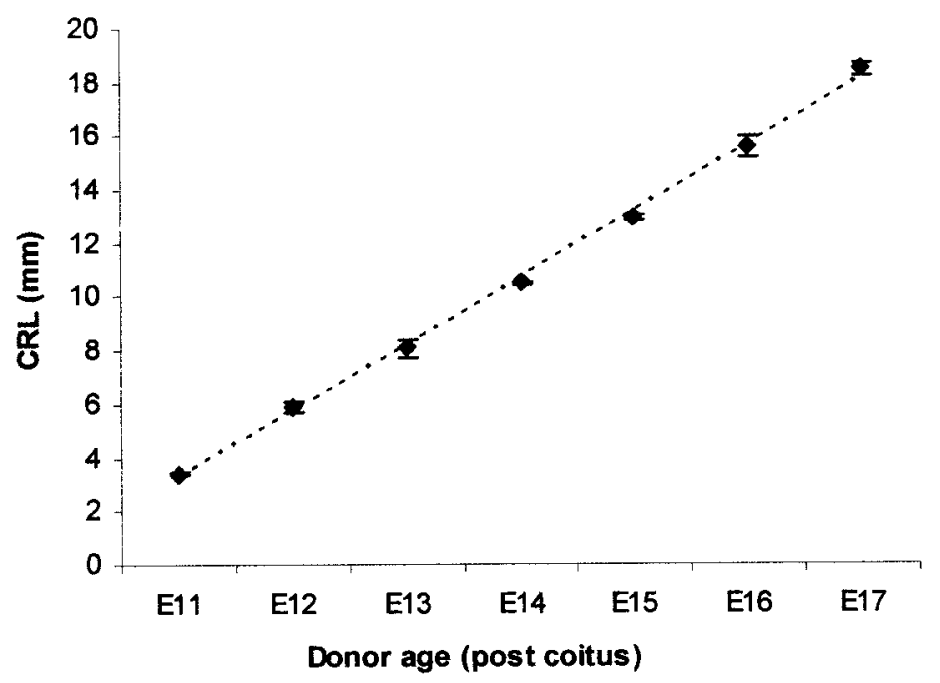

Figure 2. Plot of mean CRL in millimeters for each donor age. Error bars are SEs.

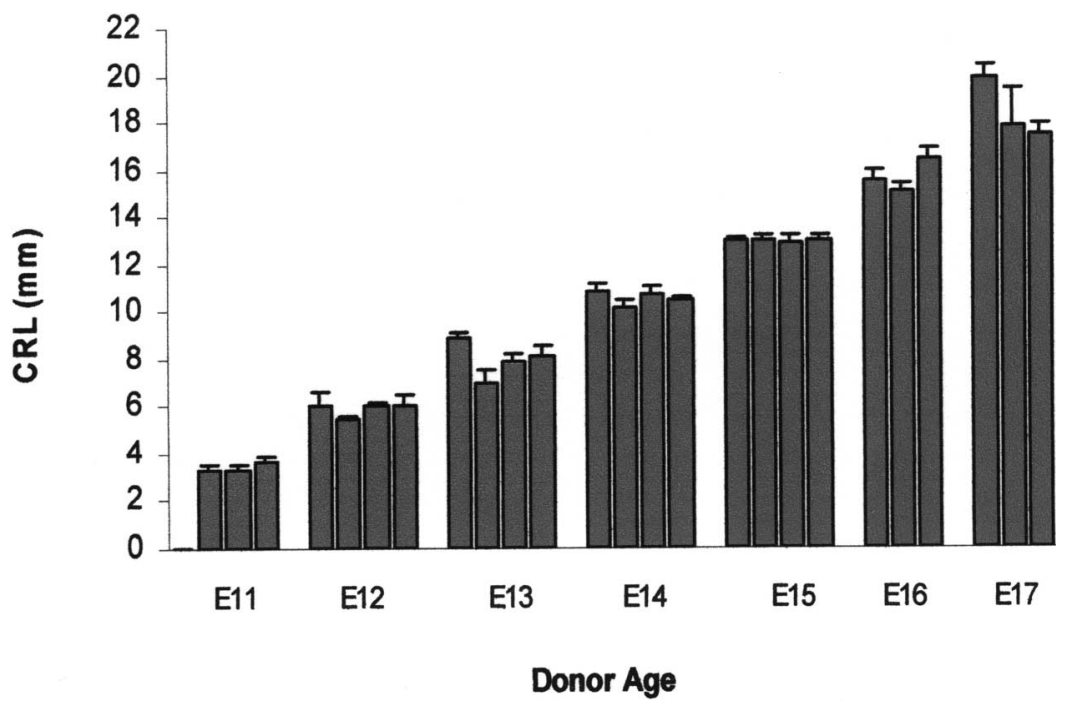

Figure 3. Plot of mean CRL against donor age for individual litters to show within-litter variation. Error bars are SEs. 


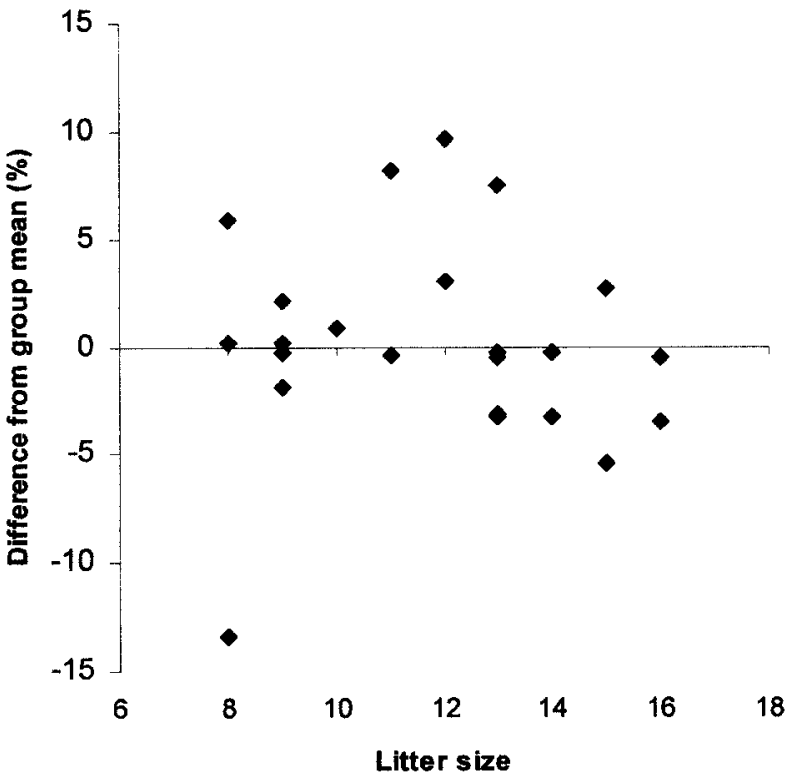

Figure 4. Plot of litter size against embryo size (expressed as a percentage variation from the mean CRL of the age group). There is no effect of litter size on the size of the embryos in the litter.

dams is normally carried out in the afternoon, and the observation of the vaginal plug is carried out in the morning, the two conventions are "out of phase" with each other by half a day. In recognition of this, some laboratories report the ages of embryos in half days; E13.5, E14.5, E15.5, etc.

Finally, in the rat neural transplantation literature, there are a number of different sources of staging information used to relate embryonic size (CRL) to embryonic age. In 1998 Ambrus et al. looked at the ontogeny of calretinin expression in embryonic rat brain (1) and produced a 9-point scale of rat development from embryonic day 12 to postnatal day 90 using fresh embryos and neonates. The Atlas for Staging Mammalian and Chick Embryos by Butler and Juurlink was published in 1987 (3). This book compares the embryonic development of the domestic fowl and 12 mammalian species including rat, mouse, and man. In this study the "greatest length" (equivalent to CRL) of embryos fixed for 2 weeks in $10 \%$ formalin was used to produce extensive staging scales for each species, and morphological characteristics were then used to relate these to the Carnegie developmental scale of human development produced in 1914 by Mall (10). In the field of Parkinson's disease neural transplantation, the staging scale most referred to is that compiled by Dunnett and Bjorklund (4), which was in turn based on the early account by Olson and colleagues in 1972 from their developmental work using rat embryonic donor tissues for intraocular transplantation (14). They used fresh embryos to modify the Butler and Juurlink data (3) and produced a 9-point scale ranging from E12 to E22. Less cited but equally informative is the paper by Lauder and Bloom describing the ontog-

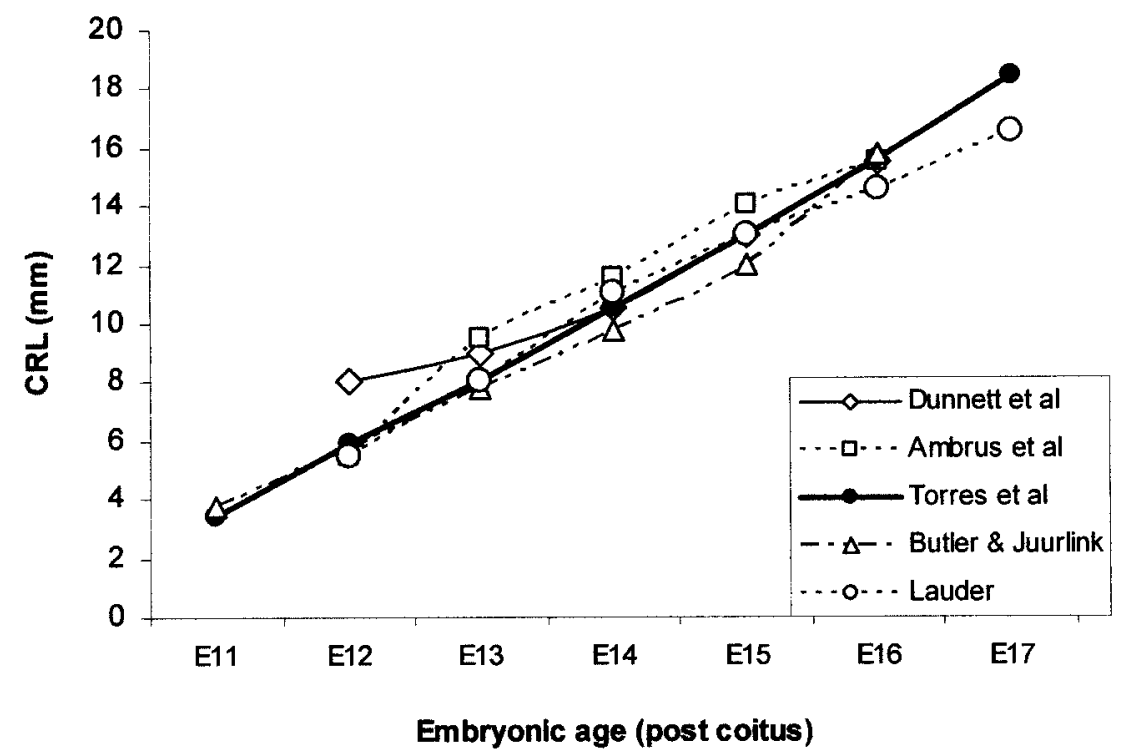

Figure 5. Comparison of the new staging scale with previous scales from the literature. All scales have been adjusted so that day 0 is the day of mating. The scale generated from the current data is shown as a solid line with the scales of Ambrus et al. (1), Butler and Juurlink (3), Dunnett and Bjorklund (4), and Lauder and Bloom (8) plotted as dashed lines for comparison. 


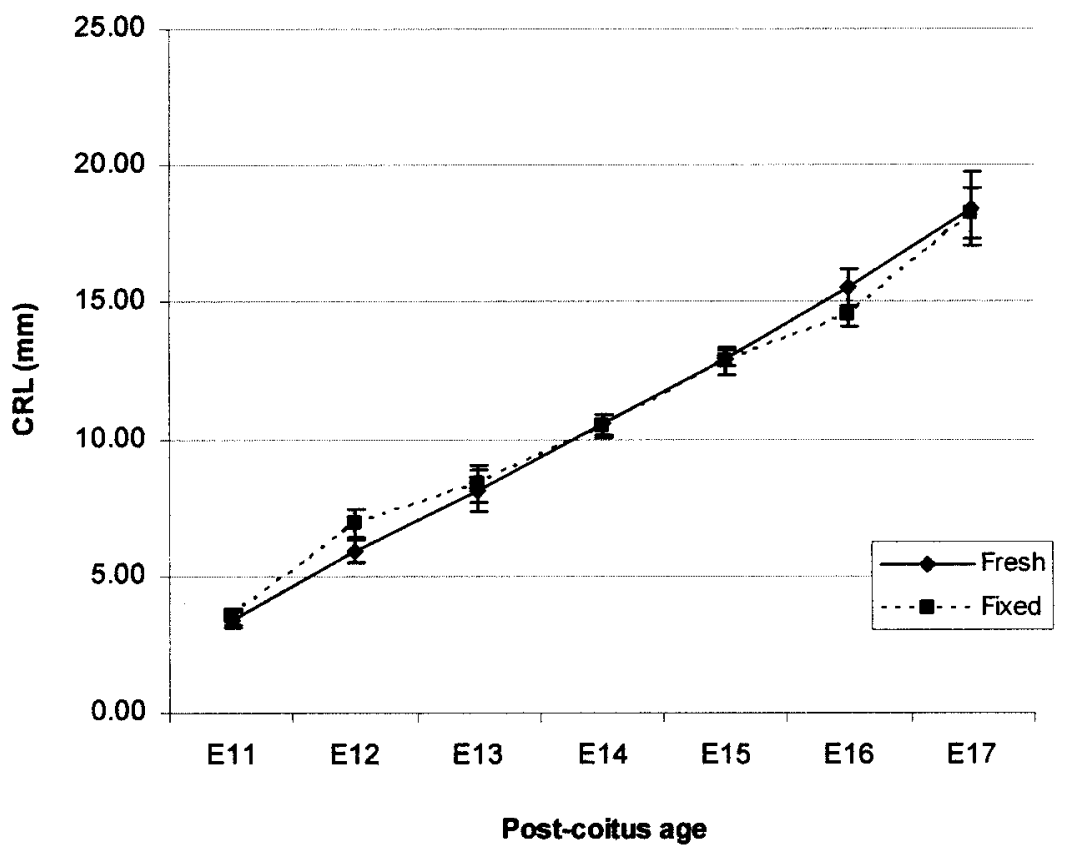

Figure 6. Comparison of the sizes of freshly collected embryos and the same specimens measured after several weeks in fixative. Fixation appears to cause slight swelling of young embryos but shrinkage of older embryos. However, overall changes are minimal and not statistically significant. Error bars are SDs.

eny of monoamine neurons in the rat midbrain in which the sizes of the embryos used are reported (8).

Figure 4 shows the staging scales obtained from these sources, plotted together with data from the current study. None of the scales concur at all points and at some ages differences in the reported CRL between scales is as much $2 \mathrm{~mm}$. The Dunnett and Bjorklund scale (4) denotes a CRL for E12 that is larger than the other four scales. Between E13 and E15 there is good correspondence between the present scale and that of Dunnett and Bjorklund (4), while CRLs are generally larger on the Ambrus et al. scale (1) and smaller on the Butler and Juurlink scale (3). The differences between scales are large enough to have a considerable influence on estimates of embryonic age. For example, an embryo with a CRL of $8 \mathrm{~mm}$ would be denoted as being aged E13 on four of the scales but would be E12 on the Dunnett and Bjorklund' scale (4), which by extrapolation would denote a 6-mm embryo as E11 or younger. Similarly, estimates of the ages of 10-, 12- and 14-mm sized embryos would differ by 1 day between the Ambrus et al. and Butler and Juurlink scales $(1,3)$.

There are a number of possible reasons for the differences seen between staging scales. Differences in the strain of rat, mating protocols, and methods of measurement used might account for most of the differences. The use of fixed embryos for the Butler and Juurlink (3) data might also be a factor. The embryos collected in the current study were remeasured following 6-12 weeks in fixative. While there was little overall effect on embryo size in this period, a significant change in the mean CRL was observed for embryos aged E12 and E16 (Fig. 6).

While for much work, the precise ageing of embryos may not be crucial, in many fields of investigation, accurate estimation of embryonic age to within a few hours may be critical. For example, in a recent paper from our laboratory, adenoviral vectors were used to deliver the differentiation factor sonic hedgehog (Shh) or the trophic factor glial cell-derived neurotrophic factor (GDNF) to embryonic grafts in a rat model of Parkinson's disease (19). Both factors were shown to improve dopamine cell yields in the grafts but had effects at different donor ages; Shh was most effective in grafts from E12 donors while GDNF had its greatest effect in E14 grafts. Transforming growth factor- $\beta$ (TGF- $\beta$ ) is another factor that has been shown to improve the survival of rat E14 dopamine grafts and it would be interesting to know whether this compound would have greater or lesser effects on grafts derived from donors of different ages (9). Similarly, the use of antiapoptotic factors such a caspase inhibitors has been shown to improve dopamine graft survival (11). Such effects may have relatively small windows of action, and future elaboration of the processes involved and the time scale of such effects will 
depend critically on an ability to define the age of the embryonic tissue employed.

Other studies have demonstrated the importance of donor age on the survival of implanted neural tissues in rat models of Huntington's disease $(5,20)$, or on the responsiveness of embryonic tissue to growth factors applied in vivo (6). The harvesting of stem cells from embryonic tissue may also dependt on donor age. In a recent paper, O'Keeffe et al. showed that in tissue cultures, donor embryos of a specific age (E12) yielded the largest number of cells and the highest yield of stems cells (13).

The current article presents an accurate staging scale for $\mathrm{CD}$ rats between the ages of E11 and E17. Embryonic development over the period studied is remarkably linear and extrapolation between the ages sampled should allow the precise ageing of any litter where the CRL can be measured accurately, assuming that growth is linear within any 24-h period (2). It should be noted that the present scale is dependent on a precise window of mating in the early morning period, at the transition from the rats' dark to light circadian cycle. There is evidence that the timing of ovulation, implantation, and first cleavage of the ovum are closely linked to the circadian cycle, and to some extent independent of the time of mating (16). Consequently, the present scale can only be advocated if a similar mating protocol is followed and may need to be revalidated for rats maintained under a different circadian cycle, or for pairings carried out as other times of day.

The data presented in this article were based on specific mating protocol in which the dams and sires were paired for just $2 \mathrm{~h}$. Charles River UK routinely use a vaginal impedance meter to identify rats in the preestrous stage of their cycle. The impedance meter simply measures resistance across the vaginal epithelium, which peaks during proestrous as the thickness of the vaginal cell wall increases. There are two such commercially available meters; the MK-10A and the EC40, made by Muramachi Kikai Co. (Japan) and Fine Science Tools Inc. (USA), respectively. Other workers have used custom-built probes and meters effectively for the same purpose $(7,15,18)$. Once selected proestrous dams can be paired at any time during the next $12 \mathrm{~h}$ for a minimum period of $2 \mathrm{~h}$ to achieve high levels of successful pregnancies.

Since completion of the current experiments we have replicated the Charles River mating protocol in-house. The vaginal impedance meter was deemed unsuitable for the purpose by Charles River UK and our early attempts in house revealed that this was indeed the case. Subsequently, we have relied on examination of cells obtained from vaginal lavage. Female, Sprague-Dawley rats maintained on a regular 12-h light/12-h dark cycle are exam- ined between 0900 and $1000 \mathrm{~h}$ by flushing the vagina with $250 \mu \mathrm{l}$ of sterile $(0.9 \%)$ saline, using a cut-down glass pipette with flame-rounded end. The collected cells are placed on a clean microscope slide and then examined without staining or cover-slipping under a light microscope. An element of diffraction is introduced by closing down the condenser diaphragm on the microscope until the cells are clearly visible. It is then a straightforward matter to determine what stage in the estrous cycle each rat is in, based on the proportions of nucleated epithelial cells, cornified cells, and inflammatory leucocytes present in each sample $(12,17)$. Samples from rats in the estrous stage of the cycle contain large numbers of cornified epithelial cells and little else. Rats in estrus are then mated for $2 \mathrm{~h}$ between 1000 and 1200 $\mathrm{h}$ and embryos collected within the same 2-h time window on the day required. In our hands, successful pregnancy is achieved in $50 \%$ of mated dams, and embryos produced in this way are at the same stage of development as those produced using the Charles River protocol. Because of the accuracy and reliability of mating we are able to meet our modest needs (maximum eight litters per month) with a stock of just 2 male and 12 female rats, with up to 4 females a week being paired. This allows time for the females from unsuccessful pairings to be identified before the next attempt, and the females from successful matings to be replaced at regular intervals.

ACKNOWLEDGMENT: This work was funded by the Medical Research Council (MRC) UK.

\section{REFERENCES}

1. Ambrus, A.; Kraftsik, R.; Barakat-Walter, I. Ontogeny of calretinin expression in rat dorsal root ganglia. Brain Res. Dev. Brain Res. 106:101-108; 1998.

2. Barbe, M. F.; Levitt, P. Age-dependent specification of the corticocortical connections of cerebral grafts. J. Neurosci. 15:1819-1834; 1995.

3. Butler, H.; Juurlink, B. H. J. An atlas for staging mammalian and chick embryos, 1st ed. Boca Raton, FL: CRC Press; 1987.

4. Dunnett, S. B.; Bjorklund, A. Basic neural transplantation techniques. I. Dissociated cell suspension grafts of embryonic ventral mesencephalon in the adult rat brain. Brain Res. Brain Res. Protoc. 1:91-99; 1997.

5. Fricker, R. A.; Torres, E. M.; Dunnett, S. B. The effects of donor stage on the survival and function of embryonic striatal grafts in the adult rat brain. I. Morphological characteristics. Neuroscience 79:695-710; 1997.

6. Kelly, C. M.; Tyers, P.; Borg, M. T.; Svendsen, C. N.; Dunnett, S. B.; Rosser, A. E. EGF and FGF-2 responsiveness of rat and mouse neural precursors derived from the embryonic CNS. Brain Res. Bull. 68:83-94; 2005.

7. Koto, M.; Miwa, M.; Togashi, M.; Tsuji, K.; Okamoto, M.; Adachi, J. [A method for detecting the optimum day for mating during the 4-day estrous cycle in the rat; measuring the value of electrical impedance of the vagina] Jikken Dobutsu. 36:195-198; 1987. 
8. Lauder, J. M.; Bloom, F. E. Ontogeny of monoamine neurons in the locus coeruleus, Raphe nuclei and substantia nigra of the rat. I. Cell differentiation. J. Comp. Neurol. 155:469-481; 1974.

9. Macauley, S. L.; Horsch, A. D.; Oterdoom, M.; Zheng, M. H.; Stewart, G. R. The effects of transforming growth factor-beta2 on dopaminergic graft survival. Cell Transplant. 13:245-252; 2004.

10. Mall, F. P. On stages in the development of human embryos from 2-25mm long. Anat. Anz. 46:78-84; 1914.

11. Marchionini, D. M.; Collier, T. J.; Pitzer, M. R.; Sortwell, C. E. Reassessment of caspase inhibition to augment grafted dopamine neuron survival. Cell Transplant. 13: 273-282; 2004.

12. Marcondes, F. K.; Bianchi, F. J.; Tanno, A. P. Determination of the estrous cycle phases of rats: some helpful considerations. Braz. J. Biol. 62:609-614; 2002.

13. O'Keeffe, G. W.; Sullivan, A. M. Donor age affects differentiation of rat ventral mesencephalic stem cells. Neurosci. Lett. 375:101-106; 2005.

14. Olson, L.; Seiger, A. Brain tissue transplanted to the anterior chamber of the eye. 1. Fluorescence histochemistry of immature catecholamine and 5-hydroxytryptamine neurons reinnervating the rat iris. Z. Zellforsch. Mikrosk. Anat. 135:175-194; 1972.
15. Ramos, S. D.; Lee, J. M.; Peuler, J. D. An inexpensive meter to measure differences in electrical resistance in the rat vagina during the ovarian cycle. J. Appl. Physiol. 91: 667-670; 2001.

16. Shalgi, R.; Kraicer, P. F. Timing of sperm transport, sperm penetration and cleavage in the rat. J. Exp. Zool. 204:353-360; 1978.

17. Solberg, P.; Evaluation of vaginal smears in the rat. http:// oslovet.veths.no/teaching/rat/oestrus/; 2004.

18. Taradach, C. Monitoring of the oestrus cycle in the rat by measurement of vaginal impedance. Arch. Toxicol. Suppl. 5:184-186; 1982.

19. Torres, E. M.; Monville, C.; Lowenstein, P. R.; Castro, M. G.; Dunnett, S. B. Delivery of sonic hedgehog or glial derived neurotrophic factor to dopamine-rich grafts in a rat model of Parkinson's disease using adenoviral vectors Increased yield of dopamine cells is dependent on embryonic donor age. Brain Res. Bull. 68:31-41; 2005.

20. Watts, C.; Dunnett, S. B.; Rosser, A. E. Effect of embryonic donor age and dissection on the DARPP-32 content of cell suspensions used for intrastriatal transplantation. Exp. Neurol. 148:271-280; 1997. 